\title{
Producción Científica en la Base de Datos Scopus de una Universidad Privada del Perú
}

\author{
José Livia ${ }^{1}$; Cesar Merino-Soto ${ }^{2}$; Rodrigo Livia-Ortiz *3

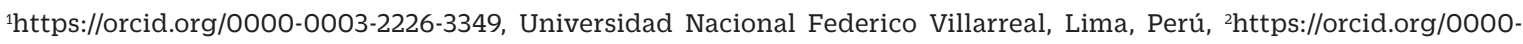 \\ 0002-1407-8306, Universidad de San Martin de Porres, Lima, Perú, ${ }^{3}$ https://orcid.org/0000-0001-5349-2596, Universidad \\ de San Martin de Porres, Lima, Perú.
}

Citar como: Livia, J., Merino-Soto, C., \& Livia-Ortiz, R. (2021). Producción Científica en la Base de Datos Scopus de una Universidad Privada del Perú. Revista Digital de Investigación en Docencia Universitaria, 16(1), e1500. https://doi.org/10.19083/10.19083/ ridu.2022.1500

Recibido: 25/07/2021. Revisado: 12/08/2021. Aceptado: 20/10/2021. Publicado: 26/11/2021

\section{Resumen}

Objetivo: evaluar la producción científica de la Universidad de San Martín de Porres (USMP) entre el periodo 1995-2020. Método: se realizó un estudio bibliométrico analizando los artículos publicados en la base de datos Scopus que registraban como afiliación del autor a la USMP, y las variables: número de publicaciones, áreas, autores, idioma, tipo de publicación, revista, colaboración y citas por trabajo. Resultados: se ubicaron un total de 880 artículos. Se evidenció una tendencia al alza en el número anual de publicaciones. La mayor parte de los artículos (43.7\%) fueron clasificados como pertenecientes al área de Medicina, seguido de Ciencias Sociales (11\%) y Psicología (7.4\%), los autores con mayor número de publicaciones fueron: Domínguez-Lara, Merino-Soto y Fujita, 61.3\% de los artículos son producidos en inglés, 58.2\% de los trabajos tienen por lo menos una cita. Conclusión: se evidencia un incremento de la producción científica con predomino del área de medicina y de artículos en inglés.

Palabras clave: bibliometría; investigación científica; universidad privada; información científica.

\section{Scientific Production of a Peruvian Private University in Scopus Database}

\begin{abstract}
Objective: to evaluate the scientific production of the Universidad de San Martin de Porres (USMP) between 1995-2020. Method: a bibliometric study was carried out, analyzing the articles published in Scopus database that registered the author's affiliation with the USMP, analyzing the variables: number of publications, areas, authors, language, type of publication, journal, collaboration, and citations by article. Results: a total of 880 articles were located. There was an upward trend in the annual number of publications. Most of the articles (43.7\%) belong to the medicine area, followed by social sciences (11\%) and psychology (7.4\%), the authors with the highest number of publications were: DomínguezLara, Merino-Soto, and Fujita, 61.3\% of the articles are produced in English, 58.2\% of the articles have at least one citation. Conclusion: there is evidence of an increase in scientific production, predominantly in the area of medicine and articles in English.
\end{abstract}

Keywords: bibliometrics; scientific production; private university; scientific information. 


\section{Introducción}

La producción científica es la esencia de las universidades (Piedra \& Martínez,2007). Es definida como la evidencia del conocimiento científico generado, permite visibilizar las investigaciones, refleja la actividad académica y científica de un investigador expresada en publicaciones que involucran a una institución o grupo social, lo cual contribuye al desarrollo del conocimiento y a la mejora en la calidad de vida de los habitantes de un país.

Una comunidad científica difunde la producción del conocimiento utilizando diversos medios: artículos, tesis, libros y ponencias (Braga et al., 2003; Tarango et al., 2015) en ese sentido, como lo señala Miguel (2011), las revistas académico-científicas son uno de los principales medios de comunicación y difusión de los resultados de las investigaciones, que promueven la institucionalización social de la ciencia y su carácter público para la evaluación de la comunidad científica.

La producción de ciencia en el mundo es evaluada a través del número de publicaciones científicas, su impacto sobre las sociedades en políticas públicas, la producción colaborativa internacional, la coautoría, el factor de impacto de la revista en la que se publica y el número de citas que tiene un artículo en la literatura científica. (Chúa \& Orozco, 2016).

Dentro de este contexto, la Superintendencia Nacional de Educación Superior Universitaria (Sunedu, 2020) publica el ranking de universidades como parte de su II informe bienal, en el que establece como indicador la producción científica de la investigación universitaria, acorde con estándares internacionales de calidad, con base en las revistas indexadas por Scopus; y ubica a la Universidad Peruana Cayetano Heredia en el primer puesto con 100 puntos y a la Universidad de San Martín de Porres en el puesto 13 con 13.39 puntos. Por otro lado, la Política de Educación Superior Técnico Productiva (Ministerio de Educación [Minedu], 2020) propone que las universidades del Perú logren un posicionamiento al ubicarse entre las 1,000 mejores universidades del mundo, aspecto vinculado con la Visión del Perú al 2030.

Por tanto, es un reto para las universidades públicas y privadas generar investigaciones $\mathrm{y}$ publicarlas en revistas indexadas de alto impac- to como las registradas en Scopus, es por ello la necesidad de estudiar la producción científica de la Universidad San Martín de Porres (USMP), institución creada en 1962 para formar profesionales. Actualmente, hay 20 carreras profesionales: administración, administración en negocios internacionales, contabilidad-finanzas, gestión de recursos humanos, psicología, medicina, enfermería, odontología, ciencias de la comunicación, derecho, economía, educación, ingeniería civil, arquitectura, ingeniería de computación-sistemas, ciencias aeronáuticas, ingeniería industrial, marketing, obstetricia y turismo - hotelería.

Según la resolución de licenciamiento (Resolución del Consejo Directivo No 024-2017-SUNEDU/CD, 2017), se reconoce que cuenta con 118 programas existentes conducentes a grado académico y 109 programas de segunda especialidad, los cuales se desarrollan en sus 11 locales ubicados en la provincia y departamento de Lima, 13 programas en su filial de Chiclayo y 12 programas en su filial Arequipa. Según el Ranking Scimago (2007-2011), la USMP ocupaba el puesto 12 (Chamana, 2013) y para el periodo 2020 ocupó el puesto 5 (De-Moya-Anegón et al., 2020).

Un trabajo efectuado sobre la producción científica de la USMP (Taype-Rondán \& Luque, 2014) refiere un total de 92 artículos en Scopus hasta el 2013, denotando una tendencia al alza en el número anual de publicaciones, con predominio de los trabajos en el área de medicina (62\%), seguido de psicología con 16.3\%; 57.6\% de los artículos fueron publicados en español, $42.4 \%$ en inglés y el $69.6 \%$ de los trabajos se realizaron en colaboración, razón por la cual la presente investigación busca actualizar la información luego de siete años del reporte de Taype-Rondán y Luque (2014) y caracterizar la producción científica de una entidad privada, en proceso de posicionamiento como una institución generadora de conocimiento científico, la misma que debe ser evaluada, con fines de diagnóstico y de monitoreo; asimismo, evaluar si los programas de investigación están dando los resultados esperados, sobre todo con la implementación de la Ley Universitaria 30220 y del licenciamiento del servicio educativo superior universitario, particularmente lo relacionado con las líneas de investigación a ser desarrolladas.

Por lo tanto, la presente investigación tiene 
como objetivos: evaluar la producción científica de la Universidad San Martín de Porres en función al número de artículos publicados entre 1995 al 2020, mediante la identificación de: a) la tendencia de las publicaciones, b) áreas de mayor producción, c) los autores con mayor número de publicaciones, d) el idioma de la publicación, e) el tipo de publicación, f) la revista de mayor publicación, g) la red de colaboración lograda, y h) artículos más citados.

\section{Método}

Se realizó un estudio bibliométrico de la producción científica de la USMP analizando los artículos publicados registrados hasta el 2020 en la base de datos Scopus editada por Elsevier. Fue elegida esta base de datos debido a que, en una inspección inicial de las publicaciones en los últimos años, se halló una fuerte tendencia de los autores por elegir revistas indizadas en esta base de datos, además de la relevancia, alcance y ventajas de la fuente de información (De Granda-Orive et al., 2011). La recuperación de la información usó la búsqueda por afiliación «Universidad San Martin de Porres» (identificador de afiliación: 60071247), filtrándose para el periodo 1995-2020. Posteriormente se descargó la base de datos en formato CSV según las variables: autores, título de documento, año, título de la fuente/revista, autores con filiaciones, resumen, tipo de documento e idioma.
La unidad de análisis fue la publicación original. El análisis estadístico se realizó por medio del SPSS 25, y Vos Viewer 1.6.1.6. Los autores garantizan la confidencialidad de los datos y que estos no serán utilizados para otros fines que no sean para la elaboración del presente trabajo. Al ser un estudio de mínimo riesgo, en donde no se realizan intervenciones, ni se obtuvieron datos de pacientes o muestras biológicas, no se requirió la autorización de un Comité de Ética Institucional.

Los aspectos estadísticos implicaron: uso del coeficiente de correlación de Pearson y la prueba t de significación de correlaciones, así como el análisis de la tendencia polinomial y exponencial, el coeficiente de determinación, análisis de frecuencia y un análisis de redes de coautoría.

\section{Resultados}

Se identificaron 880 artículos publicados en revistas científicas por la Universidad de San Martín de Porres, las mismas que se encuentran indexadas en la base de datos Scopus, siendo dos los artículos iniciales publicados en 1995 (Romero, 1995; Vojvodic \& Marroquín, 1995). De acuerdo con el año, se evidencia una tendencia al alza al número anual de publicaciones (Figura 1). El mayor número de trabajos se observó en el 2020, seguido del año 2018, habiendo un ligero descenso en el 2019.

El análisis por años permitió evaluar las tendencias de las investigaciones y se observó un in-

\section{Figura 1}

Producción científica de la USMP por año en la base de datos Scopus (1995-2020)

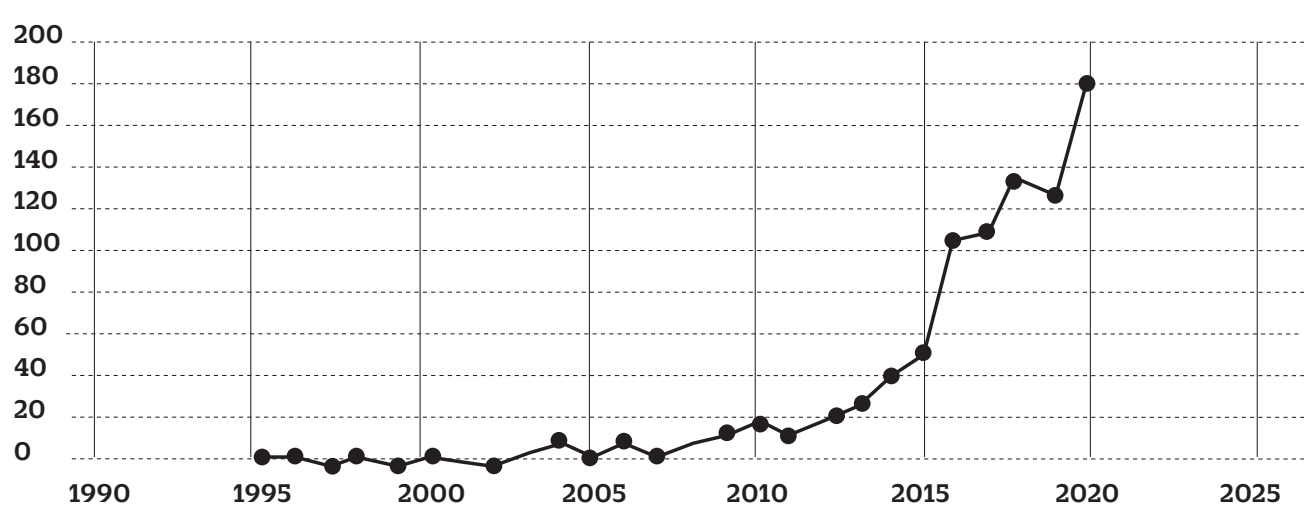


Figura 2

Frecuencia de publicación de los trabajos de grado según año de divulgación

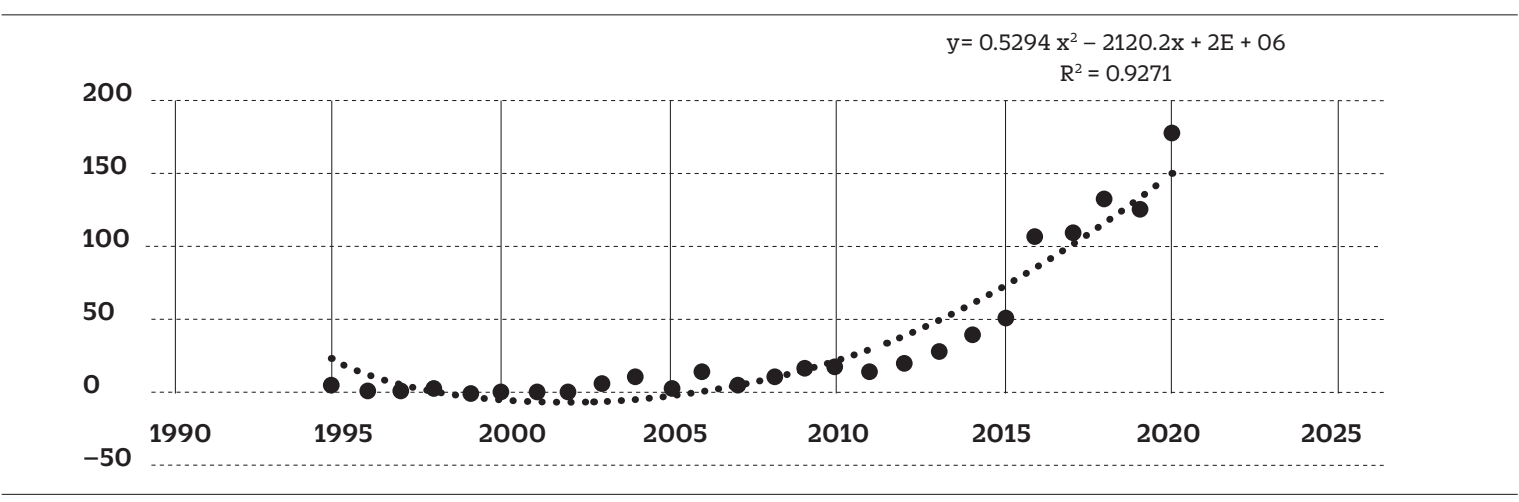

cremento gradual de las publicaciones, existiendo una correlación positiva entre el año de publicación y el número de publicaciones $(r=.80, \mathrm{p}=.01)$, lo cual constituye un indicativo del desarrollo paulatino alcanzado. El análisis de la tendencia polinomial de segundo orden señaló un coeficiente de determinación de $\mathrm{R}^{2}=92 \%$, reflejando una tendencia al incremento en la cantidad de artículos (Figura 2).

La mayor parte de los artículos fueron clasificados como pertenecientes al área de medicina (43.7\%), seguido de ciencias sociales (10.7\%), psicología (7.4\%) y bioquímica, genética y biología molecular (6.4\%), siendo el de menor número el área de energía (0.16\%) y ciencias de la decisión. (0.16\%) (Tabla 1).

\section{Tabla 1}

Frecuencia de los artículos con filiación USMP por área

\begin{tabular}{|c|c|c|}
\hline Área & $\mathbf{f}$ & $\%$ \\
\hline Medicina & 381 & $43.4 \%$ \\
\hline Ciencias sociales & 96 & $11.0 \%$ \\
\hline Psicología & 65 & $7.4 \%$ \\
\hline Bioquímica, genética y biología molecular & 55 & $6.4 \%$ \\
\hline Odontología & 42 & $4.8 \%$ \\
\hline Ciencias computación & 28 & $3.2 \%$ \\
\hline Enfermería & 27 & $3.1 \%$ \\
\hline Agricultura y ciencias biológicas & 24 & $2.7 \%$ \\
\hline Inmunología y microbiología & 21 & $2.4 \%$ \\
\hline Farmacología, toxicología y farmacia & 17 & $1.9 \%$ \\
\hline Negocios, administración y contabilidad & 16 & $1.8 \%$ \\
\hline Ingeniería & 16 & $1.8 \%$ \\
\hline Neurociencias & 11 & $1.3 \%$ \\
\hline Economía, econometría y finanzas & 11 & $1.3 \%$ \\
\hline Multidisciplinario & 11 & $1.2 \%$ \\
\hline Física y astronomía & 11 & $1.2 \%$ \\
\hline Artes y humanidades & 10 & $1.1 \%$ \\
\hline Ciencias de la salud & 7 & $0.8 \%$ \\
\hline Química & 6 & $0.7 \%$ \\
\hline Ciencias materiales & 6 & $0.7 \%$ \\
\hline Ciencias del ambiente & 5 & $0.6 \%$ \\
\hline Matemática & 5 & $0.6 \%$ \\
\hline Ingeniería química & 3 & $0.3 \%$ \\
\hline Ciencias de la tierra y planetarias & 3 & $0.3 \%$ \\
\hline Ciencias de la decisión & 2 & $0.2 \%$ \\
\hline Energía & 2 & $0.2 \%$ \\
\hline Total & 880 & $100.0 \%$ \\
\hline
\end{tabular}




\section{Tabla 2}

Frecuencia de los artículos con filiación USMP por área

\begin{tabular}{ccc}
\hline Posición & Autores & $\boldsymbol{f}$ \\
\hline 1 & Merino-Soto, C. & 61 \\
2 & Domínguez-Lara, S.S. & 53 \\
3 & Domínguez-Lara, S.A. & 50 \\
4 & Fujita, R. R. & 36 \\
5 & Taype-Rondan, A.A. & 27 \\
6 & Sánchez, S.E. & 25 \\
7 & Díaz-Vélez, C. & 23 \\
8 & Gelaye, B. & 21 \\
9 & Parodi, J.F. & 21 \\
10 & Rossell-Perry, P. & 21 \\
11 & Runzer-Colmenares, F.M. & 20 \\
12 & Morales-Vadillo, R. & 18 \\
13 & Toro-Huamanchumo, C.J. & 18 \\
14 & Sandoval, J.R. & 17 \\
15 & Urrunaga-Pastor, D. & 17 \\
16 & Alvarez-Risco, A. & 15 \\
17 & Huarcaya-Victoria, J. & 15 \\
19 & Benites-Zapata, V.A. & 14 \\
20 & Fernández-Arata, M. & 13 \\
\hline
\end{tabular}

Respecto de los cuatro autores más productivos se identifica a Merino-Soto (61 documentos), Domínguez-Lara (53 documentos), Domínguez-Lara, S.A. (50 documentos) y Fujita (36 documentos), observándose en la tabla 2 los primeros 20 con mayor cantidad de trabajos.

La coautoría refleja la colaboración científica y los vínculos entre los investigadores pudién- dose identificar los autores que trabajan en red y los que trabajan en forma individual. La figura 3 muestra el mapa de la red de coautorías, observándose los autores más productivos y sus vínculos. Los colores indican agrupaciones de investigadores que están relativamente relacionados entre sí, identificándose cinco grupos de colaboración bien definidos.

\section{Figura 3}

Redes de coautoría de los investigadores con filiación USMP

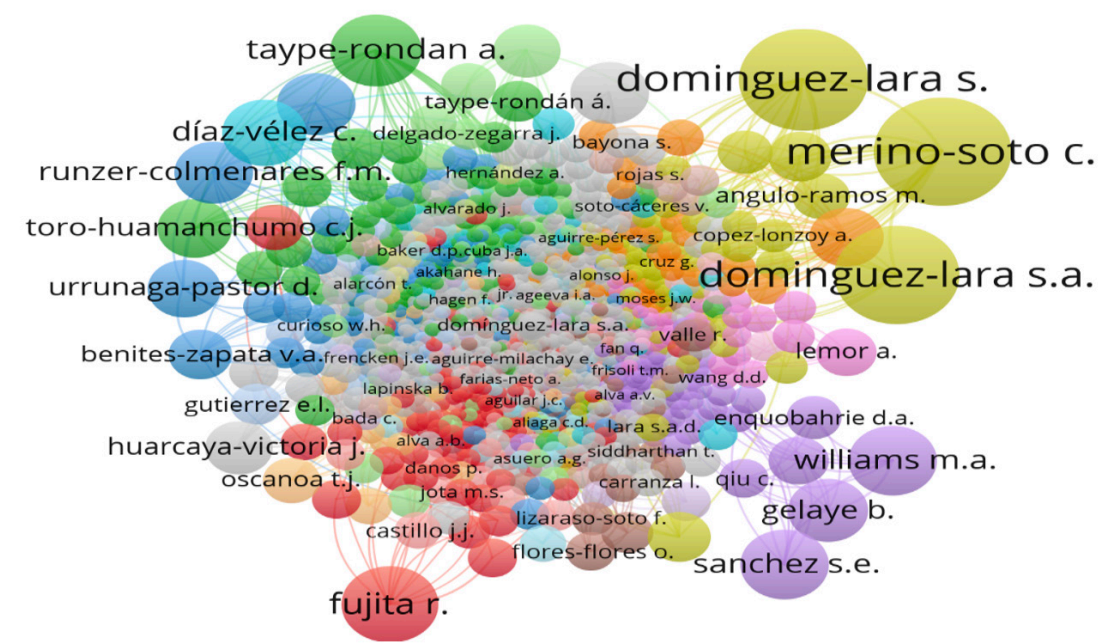




\section{Figura 4}

Vínculos de los investigadores por países

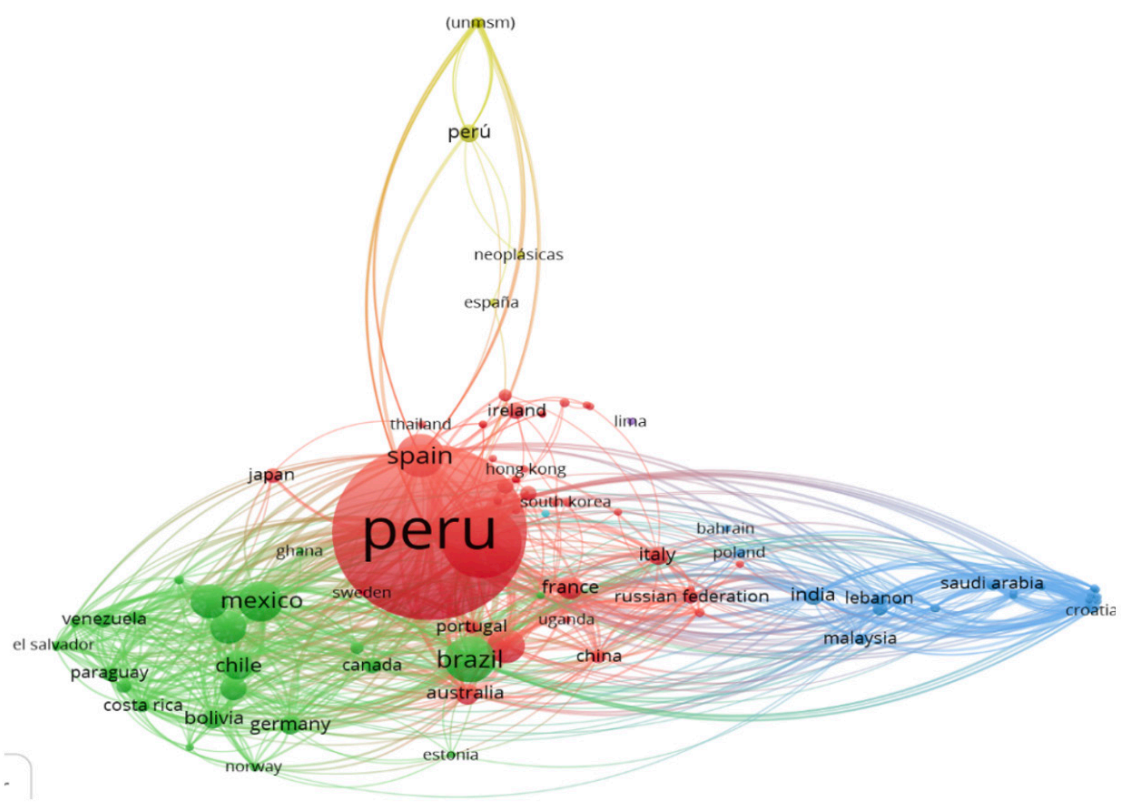

Respecto de los vínculos de los investigadores, de acuerdo con los países, se puede observar tres clústeres de vínculos, uno interno con instituciones peruanas (hospitales y universidades), un grupo externo conformado por Venezuela, México, Chile, Brasil, Paraguay y El Salvador, otro grupo de vínculos con China, España, Francia, Rusia, Italia, Portugal, así como otro grupo Arabia Saudita, Croacia, Malasia, India, Polonia y Líbano. (Figura 4).
Respecto del idioma en que son publicados los artículos el 61,3\% está en inglés, el 38.3\% en español y 0,5\% en portugués. Al inicio hubo una ligera presencia de trabajos en español para luego mantener un predominio del inglés.

En relación con los artículos por tipo de documento el 68,5\% fueron artículos originales, seguidos de cartas al editor con el 18\% y de revisión con 6.5\%. (Tabla 3).

Tabla 3

Publicaciones en la base de datos Scopus por tipos de documento

\begin{tabular}{ccc}
\hline Tipo documento & $f$ & $\%$ \\
\hline Artículo & 596 & $67.7 \%$ \\
Carta & 164 & $18.6 \%$ \\
Revisión & 57 & $6.5 \%$ \\
Documento de sesión & 30 & $3.4 \%$ \\
Capítulo de libro & 11 & $1.3 \%$ \\
Nota & 9 & $1.0 \%$ \\
Editorial & 8 & $0.9 \%$ \\
Erratum & 2 & $0.2 \%$ \\
Libro & 1 & $0.1 \%$ \\
Encuesta corta & 1 & $0.1 \%$ \\
Indefinido & 1 & $0.1 \%$ \\
Total & 880 & $100 \%$
\end{tabular}


Tabla 4

Revistas con mayor frecuencia de publicaciones de la USMP

\begin{tabular}{cc}
\hline Tipo documento & $\boldsymbol{f}$ \\
\hline Revista Peruana de Medicina Experimental & 59 \\
Educación médica & 33 \\
Revista Médica de Chile & 20 \\
Revista de Gastroenterología & 18 \\
Journal of Oral Research & 11 \\
Salud Pública de México & 11 \\
\hline
\end{tabular}

Figura 5

Producción científica en Scopus del área de medicina de la USMP (1995-2020)

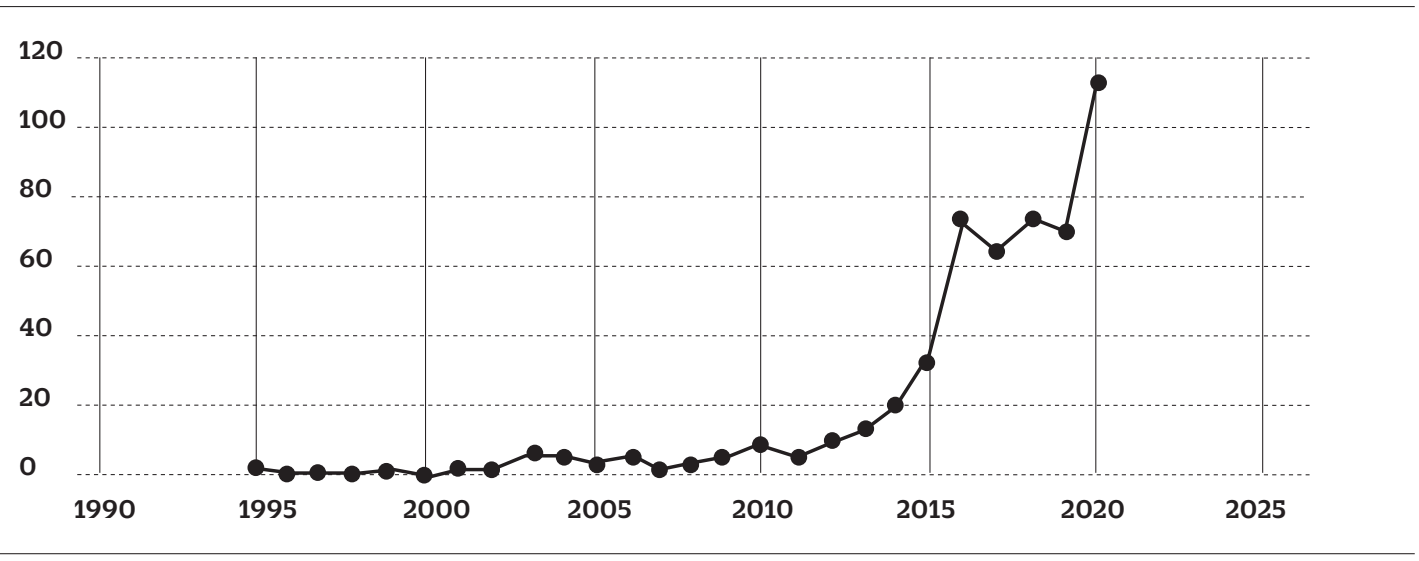

En lo que respecta a las citas, se observa que el 41.8\% de los artículos no tienen ninguna cita, en cambio el trabajo de Garred et al. (2006) tiene 307 citas, seguido de la investigación de NCD Risk Factor Collaboration (NCD-RisC, 2019) con 148 citas y la publicación de Knowles et al. (2011) con 108 citas.

Las cinco revistas donde hay mayor predominio de artículos con filiación USMP son: Revista Peruana de Medicina Experimental y Salud Pública de Perú, seguido de Educación Médica de España, Revista Médica de Chile, Revista de Gastroenterología de Perú, Journal of Oral Research de Chile y Salud Publica de México (Tabla 4).

Respecto del área de medicina se identificaron 519 artículos, observándose una tendencia de crecimiento progresivo, excepto un periodo de inestabilidad (2015-2020), siendo los años más productivos el 2016 y el 2020. (Figura 5).

El análisis por años permitió evaluar las tendencias de las investigaciones en medicina y se observó un incremento gradual de las publicaciones, El análisis de la tendencia polinomial de segundo orden, señaló un coeficiente de determinación de R2=89\%, reflejando una tendencia al incremento en la cantidad de artículos (Figura 6).

En el área de medicina, los cinco autores más productivos fueron Domínguez-Lara, Merino-Soto, Sánchez, Tapie, Rosell y Gelaye (Tabla 5).

El análisis del área de ciencias sociales identificó a 131 artículos mostrando una tendencia creciente con un ligero descenso los años 2016 y 2019, siendo los años más productivos el 2018 y el 2020 (Figura 7).

El análisis por años permitió evaluar las tendencias de las investigaciones en ciencias sociales y se observó un incremento gradual de las publicaciones. El análisis de la tendencia polinomial de segundo orden señaló un coeficiente de determinación de R2=84\%, reflejando una tendencia al incremento en la cantidad de artículos (Figura 8). 
Figura 6

Curva polinomial de la producción científica en medicina

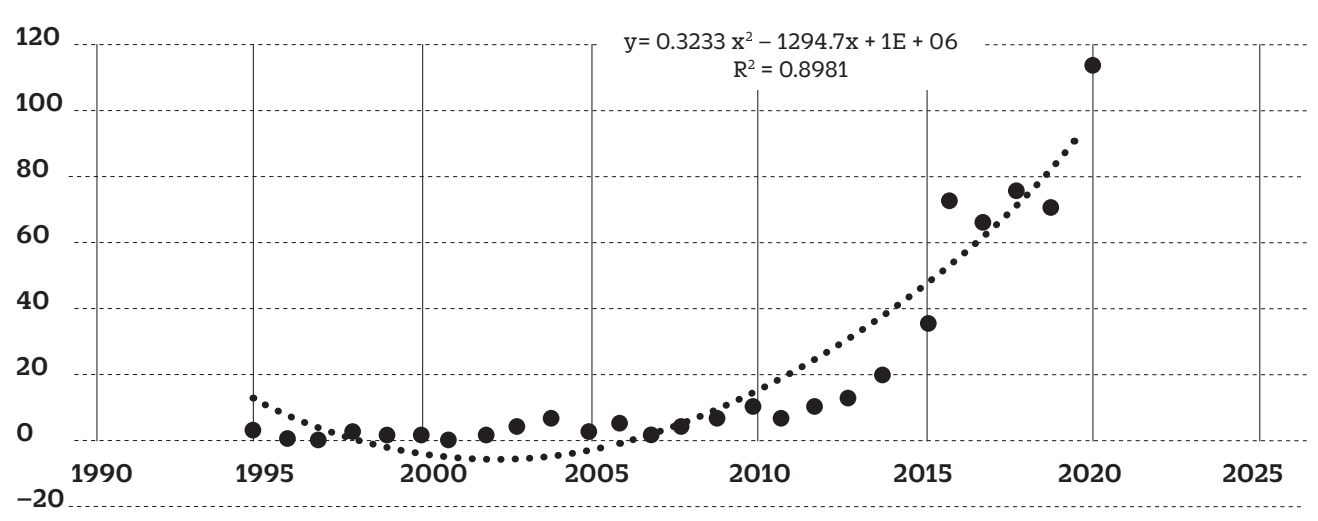

\section{Figura 7}

Producción científica en Scopus del área de ciencias sociales de la USMP (1995-2020)

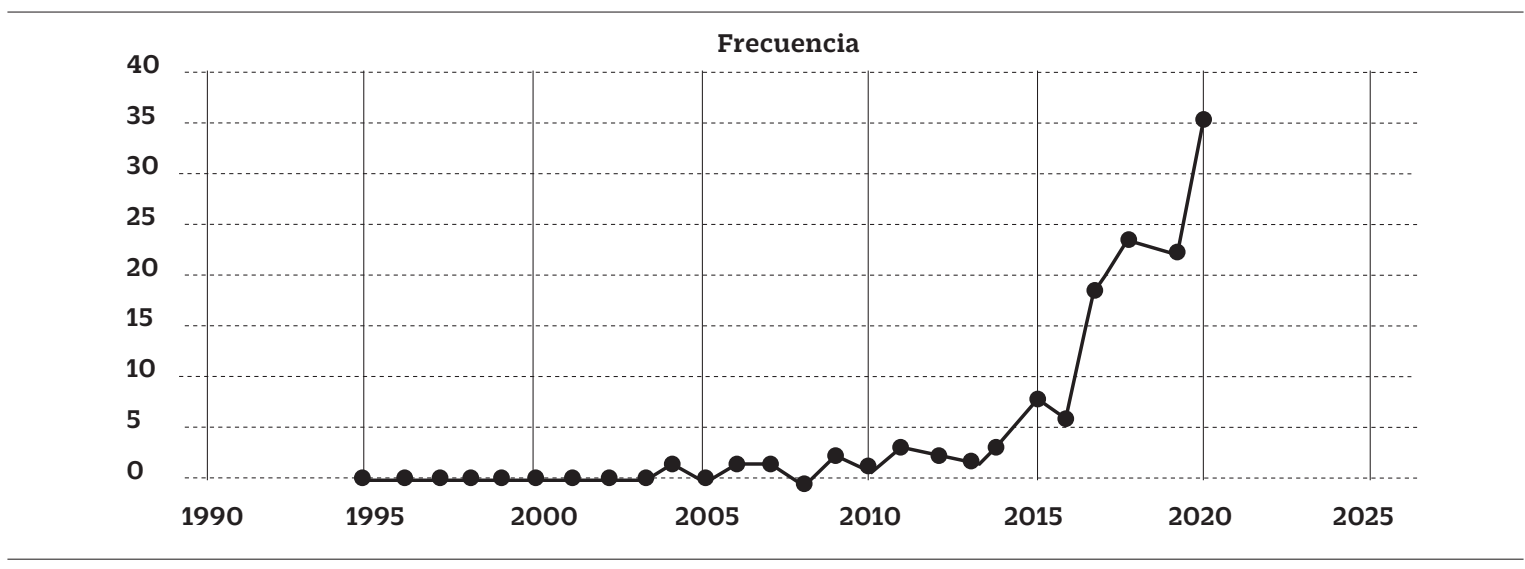

Tabla 5

Diez autores más productivos en el área de medicina de la USMP

\begin{tabular}{cc}
\hline Autores & $\boldsymbol{f}$ \\
\hline Domínguez-Lara, S.A. & 33 \\
Merino-Soto, C. & 25 \\
Sánchez, S.E. & 22 \\
Taype-Rondan, A. & 22 \\
Rossell-Perry, P. & 20 \\
Gelaye, B. & 19 \\
Parodi, J.F & 18 \\
Runzer-Colmenares, F.M. & 18 \\
Díaz-Vélez, C. & 17 \\
Fujita, R. & 17 \\
\hline
\end{tabular}


Figura 8

Curva polinomial de la producción científica en ciencias sociales

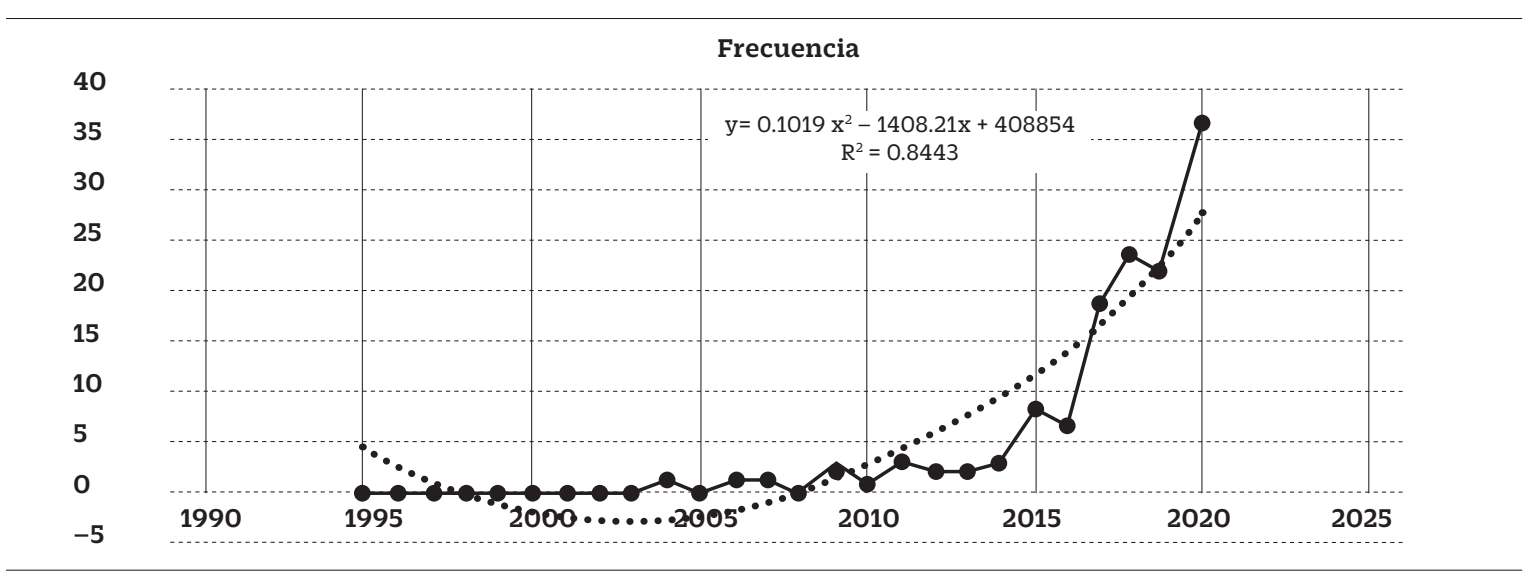

Figura 9

Producción científica del área de psicología

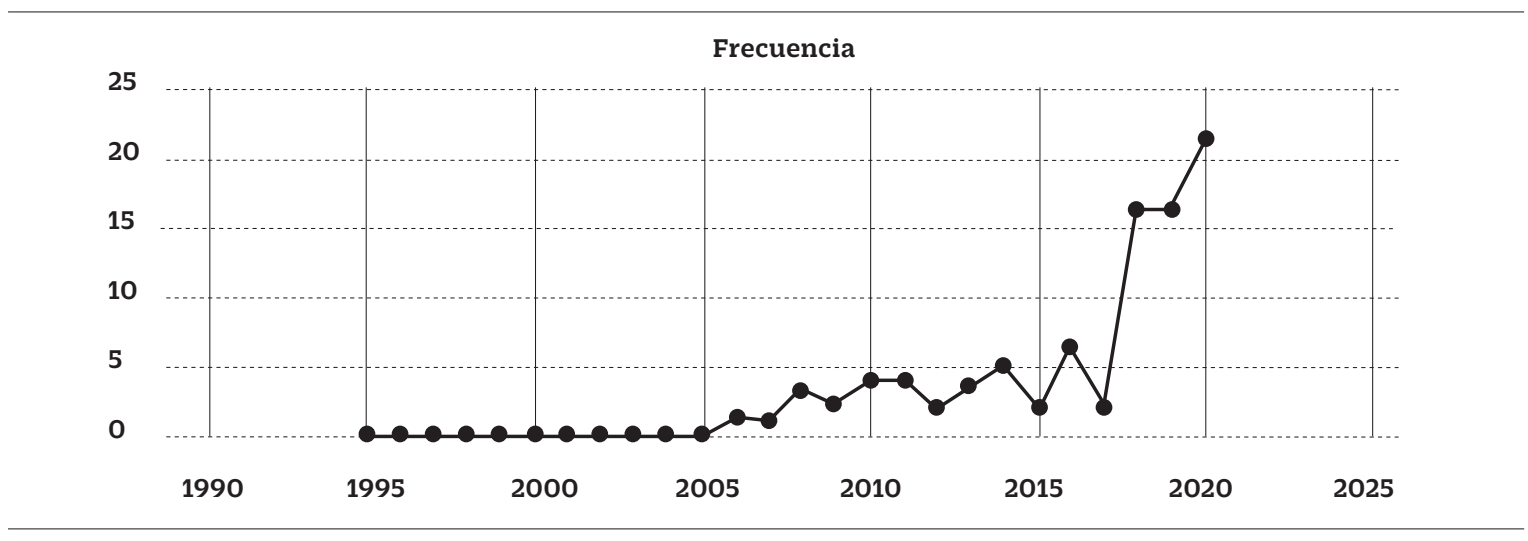

Tabla 6

Producción científica por autores en el área de ciencias sociales

\begin{tabular}{cc}
\hline Autores & $\boldsymbol{f}$ \\
\hline Merino-Soto, C. & 18 \\
Domínguez-Lara, S. & 16 \\
Domínguez-Lara, S.A. & 11 \\
Fernández-Arata, M. & 6 \\
Toro-Huamanchuco, C.J. & 6 \\
Parodi, J. F. & 5 \\
Apaza, C.R. & 4 \\
Díaz-Vélez, C. & 4 \\
Garay-Argandoña, R. & 4 \\
Navarro-Loli, J. S. & 4
\end{tabular}

En cuanto a los cinco autores con mayor producción científica en ciencias sociales fueron: Merino-Soto, C., Domínguez-Lara, S., Domínguez -Lara, S.A. y Fernández-Arata, M. (tabla 6).
En el área de psicología, se ubicaron 89 documentos con una producción muy irregular logrando un crecimiento significativo, siendo los años 2018, 2019 y 2020 los más productivos (Figura 9). 
Figura 10

Curva polinomial de la producción científica en ciencias sociales

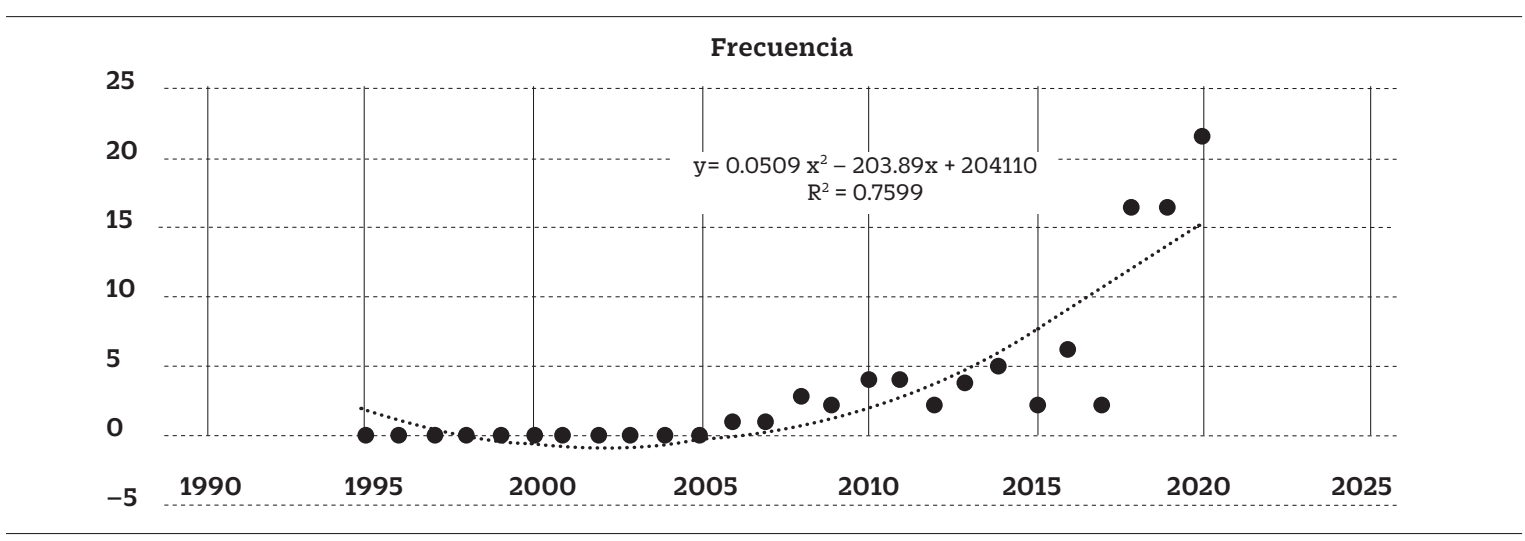

Tabla 7

Autores con mayor producción en el área de ciencias psicología

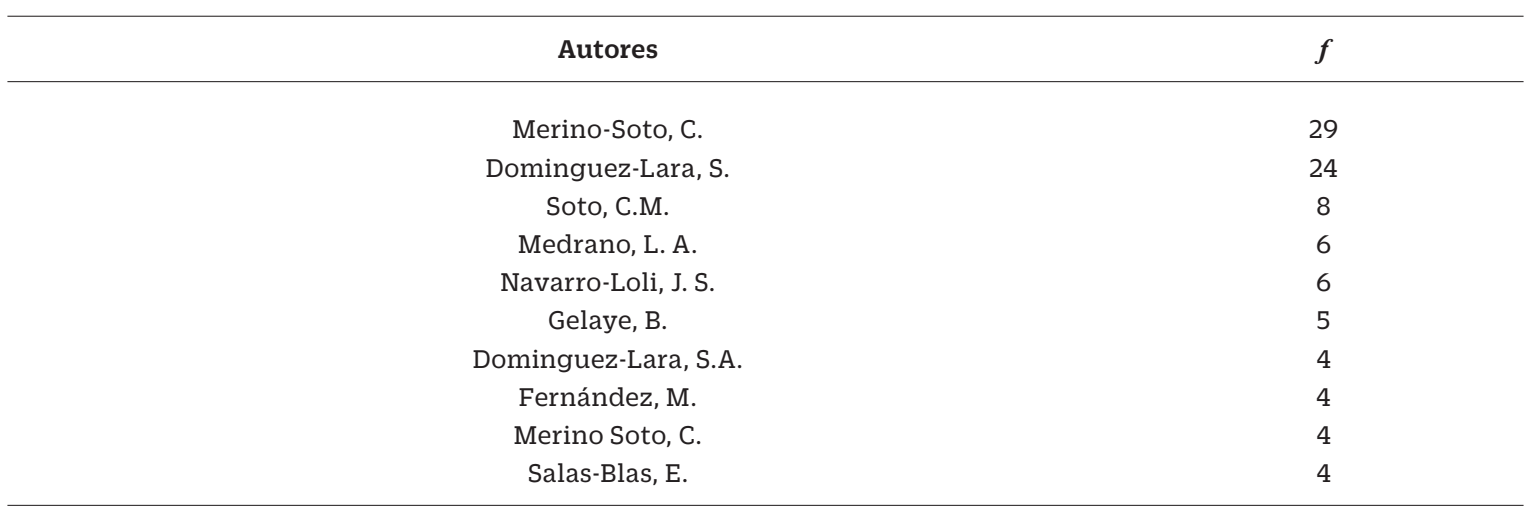

El análisis por años permitió evaluar las tendencias de las investigaciones en psicología y se observó un incremento gradual de las publicaciones, El análisis de la tendencia polinomial de segundo orden señaló un coeficiente de determinación de R2=75\%, reflejando una tendencia al incremento en la cantidad de artículos (Figura 10).

En lo que se refiere a los cinco autores con mayor producción científica en psicología fueron: Merino-Soto, Domínguez -Lara, S, Domínguez -Lara SA y Fernández-Arata (Tabla 7).

Con relación al área de bioquímica se ubican 76 publicaciones con una producción irregular y con un descenso significativo en el último año, logrando la mayor producción el 2019 (Figura 11).

El análisis por años permitió evaluar las tendencias de las investigaciones en bioquímica y se observó un incremento gradual de las publicaciones. El análisis de la tendencia polinomial de segundo orden señaló un coeficiente de determinación de $\mathrm{R} 2=81 \%$, reflejando una tendencia al incremento en la cantidad de artículos (Figura 12).

Los cinco autores con mayor producción científica en bioquímica fueron: Fujita, Sandoval, Acosta y Santos (Tabla 8).

\section{Discusión}

La bibliometría permite analizar la producción científica y es considerada como la aplicación de los análisis estadísticos para evaluar las características del uso y creación de documentos, sus productores y consumidores, de tal forma que permite observar el estado de la ciencia y la tecnología, a través de la producción global de la literatura científica en un nivel dado de especialización (Solano et al., 2009). 
Figura 11

Producción científica en Scopus en el área de bioquímica

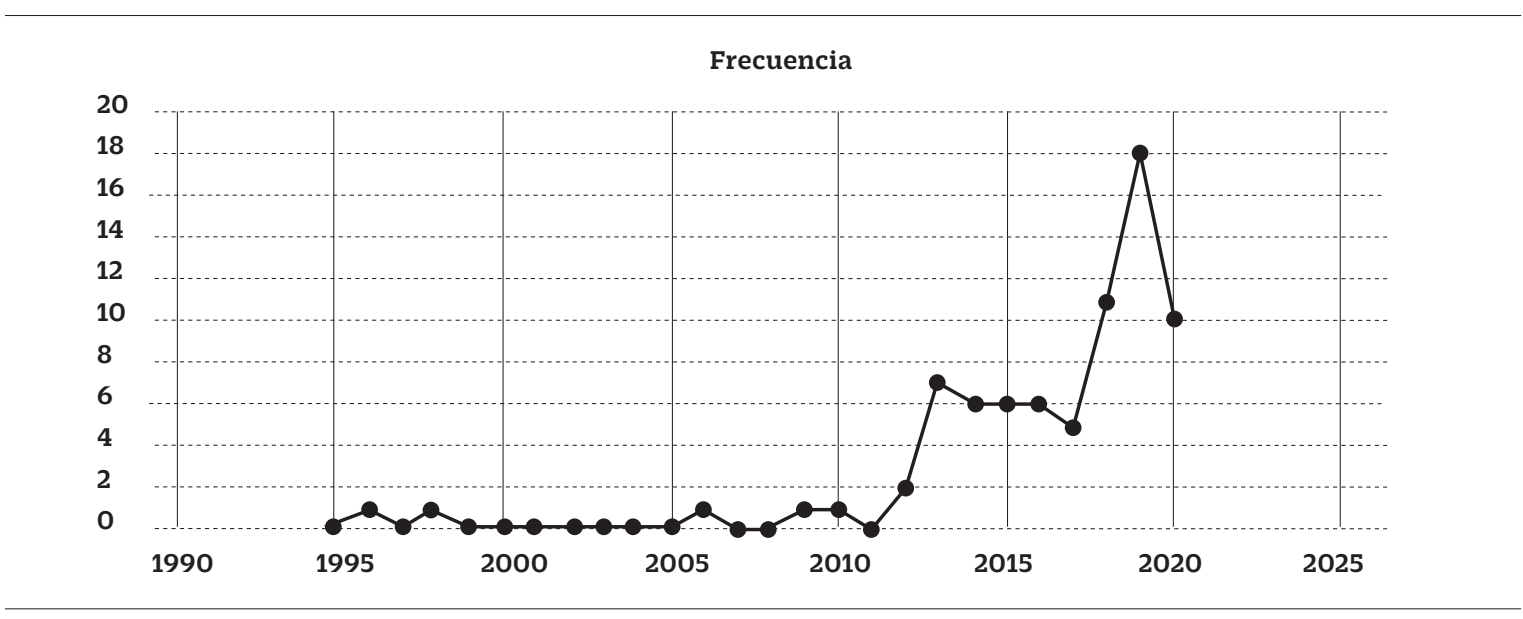

Figura 12

Curva polinomial de la producción científica en el área de bioquímica.

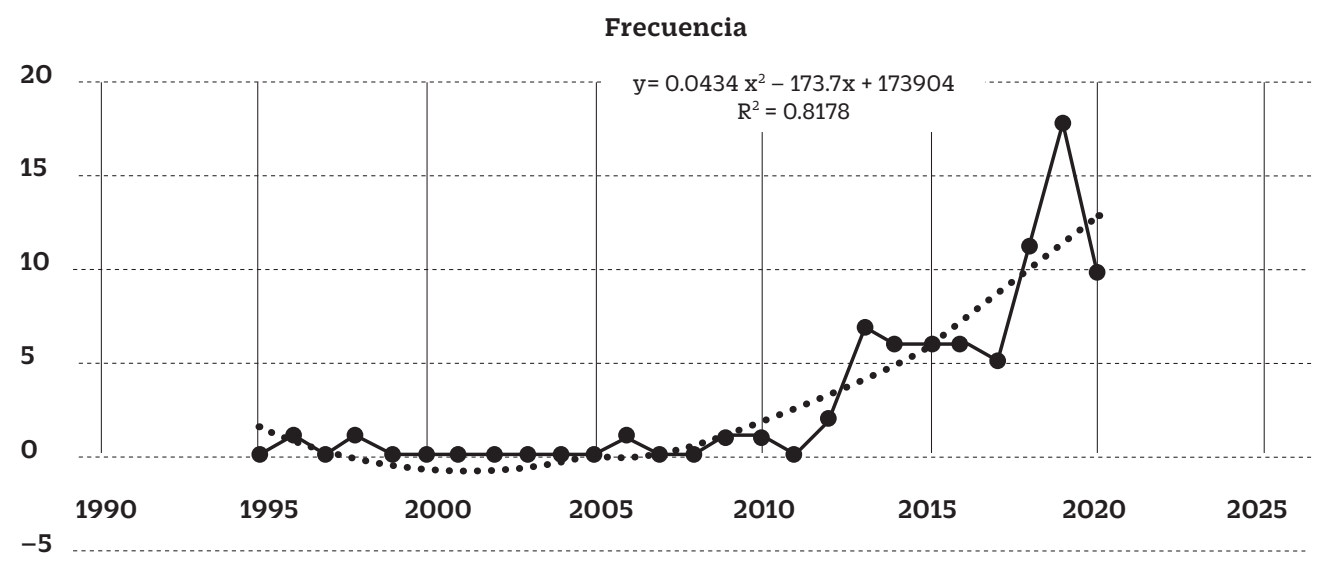

Tabla 8

Autores con mayor producción científica en el área de bioquímica

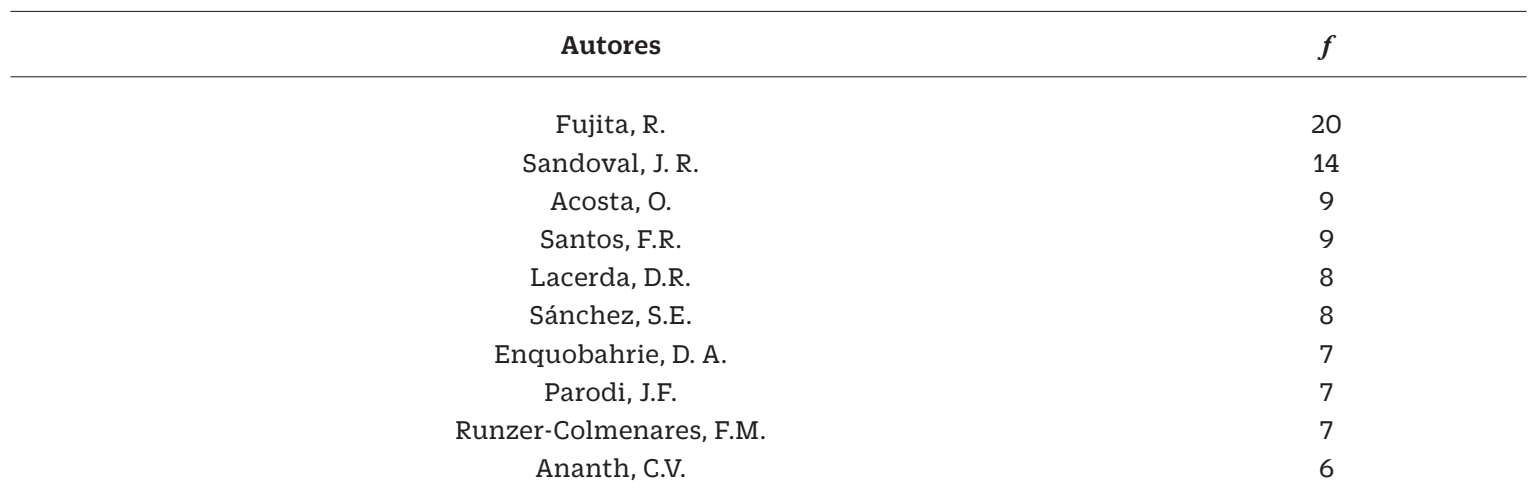


Por tal razón se aplicó esta metodología a la producción científica de la Universidad San Martin de Porres cuyos trabajos se encontraban registrados en Scopus. La USMP fue la sexta universidad privada creada en el Perú en 1962 y ha incrementado su producción científica, por lo cual fue necesario evaluarla, debido a su creciente reconocimiento en su productividad científica.

La producción científica de la USMP denota una tendencia de crecimiento polinómica logrando 880 documentos desde 1995 al 2020. Si comparamos la media de trabajos entre 1995-2013, que fue el rango de años del estudio de Taype-Rondan y Luque (2014) y la media de trabajos publicados entre el 2014-2020, se observa una diferencia de 100 artículos entre los promedios ( $\bar{x}=8.9$ versus $\bar{x}=106.5$ ), lo cual también se refleja en los acumulados; en el primer periodo señalado de 19 años sumaron 134 artículos y en el segundo periodo de siete años el acumulado fue de 746, incremento que le ha permitido ocupar a la USMP el quinto lugar en el Ranking Scopus 2020, al reflejar el crecimiento progresivo exponencial.

El área de mayor producción fue medicina, casi la mitad de la producción pertenece a dicho sector del conocimiento, con un aumento sostenido de las investigaciones. Este aspecto también se observa cuando se efectúa un análisis de la producción científica del Perú, el área de medicina al 2020 alcanza 1,819 documentos en Country Ranking Scimago Journal (https://www.scimagojr.com/countrysearch.php?country=PE), aspecto corroborado por Limaymanta et al. (2020), quienes señalan que en el Perú entre las 10 primeras disciplinas con mayor producción se ubican las ciencias médicas. Asimismo, las otras áreas de mayor crecimiento en la USMP son ciencias sociales, psicología y bioquímica, sectores que los gestores de la universidad deben fortalecer, al igual que medicina que ha descendido, de representar el 68\% de los artículos (Taype-Rondán \& Luque, 2014) a un $43 \%$ de la producción total. Se debe señalar que la educación médica exige que el profesional médico y de las ciencias de la salud sea formado integralmente desarrollando competencias específicas y genéricas, destacando dentro de las primeras las áreas o dominios de los fundamentos científicos y la investigación y aplicación del método científico (Risco de Domínguez, 2007).
La mayor presentación de trabajos recae en tres autores: Domínguez-Lara (103 trabajos), Merino-Soto (74 trabajos) y Fujita (36 trabajos), llamando la atención la falta de normalización de las firmas de los dos primeros autores, aspecto que tiene como alternativa el ORCID, como identificador único del investigador (Perilla-Rodríguez \& Pérez-Acosta, 2016). Una implicación de esto es que, la normalización del nombre de los autores en investigadores emergentes debe ser explícito, y promovido como una posible política universitaria entre investigadores en todos los niveles.

Otra variable de análisis fue el idioma de publicación, siendo la mayor cantidad de los artículos en inglés, seguido del español. Al respecto, Niño-Puello (2013) señala que el inglés no es el idioma más hablado en el mundo, pero sí el más importante y sostiene que adquiere significación en las publicaciones científicas desde la Revolución Industrial, considerándose como la lengua franca de la comunidad científica. Solo para indicar que el $80 \%$ de las revistas especializadas indexadas en Scopus son publicadas en inglés, y, por lo tanto, su relevancia para promoción de la investigación y el incremento de las citaciones no se pone en cuestionamiento. Para el presente caso se observa que todas las publicaciones han sido en colaboración.

Respecto a los tipos de trabajos se observa que predominan los artículos científicos seguido de cartas al editor y publicaciones de revisión, representando el 93\% de la producción científica. Los resultados evidencian que el vehículo de comunicación de la ciencia es el artículo empírico, que expresan ideas y hallazgos originales (Jiménez, 2015); también se destacan las cartas al editor, que establecen un aspecto interactivo entre los investigadores reflejando el juicio crítico (Fernández, 2014) y aportes novedosos para el contexto en que se publican. Muchas de las cartas al editor publicadas en los últimos años han consistido en dos tipos de contenidos: observaciones críticas sobre los análisis realizados, y con consecuente reanálisis reportado en la misma carta, y procedimientos analíticos interesantes. Como nota adicional, fue poco frecuente hallar respuestas formales a las cartas generadas por los autores.

Las redes de colaboración permiten estudiar los grupos de investigación, pero sobre todo la 
colaboración científica permitiendo profundizar líneas de investigación y medir la producción colectiva de conocimiento. Los resultados indicaron cinco grupos, uno liderado por Domínguez-Lara que unido a Merino-Soto, representan autorías en psicología y artículos en revistas de ciencias médicas, con vínculos entre ellos, donde ambos lograrían la mayor hegemonía en la USMP; otro grupo liderado por Fujita de bioquímica, Taype-Rondan y un grupo más disperso de Williams y Sánchez. La visualización de redes de colaboración refleja la fuerte tendencia de las líneas en las ciencias naturales. También se observa las relaciones internacionales entre los autores, aspecto que se refleja en otros estudios (Huamaní \& Mayta-Tristán, 2010) donde se indica que las instituciones peruanas más productivas colaboran más intensamente con instituciones extranjeras que con nacionales.

Esta investigación no cubre toda la producción científica de los investigadores de la USMP, es probable que se puedan ubicar otros trabajos en el Web of Science y Scielo, así como otras bases de datos, pero esta contribución proporciona información suficiente para futuras investigaciones que permitan evaluar otros indicadores bibliométricos, así como evaluar el impacto de la implementación de las condiciones básicas de calidad implementadas por la Sunedu.

Se ha observado un crecimiento de la producción científica en la USMP y se espera siga siendo sostenido, que involucre otras áreas de la universidad y potencie las comunidades que se van consolidando (medicina, ciencias sociales y psicología), desarrollándose una política de incentivos. Como expresan Astorne y Lepage (2011) actualmente las cualificaciones están enfocadas en educar e investigar. Lo expresado va acorde con la Política Nacional de Educación Superior establecida por el Minedu (2020), que tiene como objetivo que la educación superior cumpla un rol clave en el desarrollo y la competitividad del país, así como lograr que al año 2030 diez universidades peruanas se puedan ubicar entre las 1,000 mejores en el ámbito mundial.

\section{Referencias}

Astorne, R., \& Lepage, C. (2011). La formación universitaria en investigación aplicada y el contexto de la complejidad: una perspectiva estratégica para la innovación en ingeniería. Revista Digital de Inves tigación en Docencia Universitaria, 5(1), 66-91. https://doi.org/10.19083/ridu.5.6

Braga, C., Malerbo, M. B., \& Silva, M. R. (2003). Errores en las referencias bibliográficas de la producción académica: un estudio de caso. Scire: Representación y Organización del Conocimiento, 9(1), 133 138. https://ibersid.eu/ojs/index.php/scire/article/ view/1461

Chamana, R. A. (2013). Revisión y evaluación de las publicaciones científicas de la USMP. Universidad de San Martin de Porres. https://repositorio.usmp.edu. pe/bitstream/handle/20.500.12727/1198/chamana_ rr.pdf?sequence=1\&isAllowed=y

Chúa, C., \& Orozco, R. (2016). La producción científica. Revista médica (Colegio de Médicos y Cirujanos de Guatemala), 155(1), 7-13. https://doi.org/10.36109/rmg. v155i1.26

De Granda-Orive, J. I., Alonso-Arroyo, A., \& Roig-Vázquez, F. (2011). ¿Qué base de datos debemos emplear para nuestros análisis bibliográficos? Web of Science versus SCOPUS. Archivos de Bronconeumología, 47(4), 213-217. https://doi.org/10.1016/j.arbres.2010.10.007

De-Moya-Anegón, F., Herrán-Páez, E., Bustos-González, A., Corera-Álvarez, E., Tibaná-Herrera, G., Rivadeneyra, F. (2020). Ranking iberoamericano de instituciones de educación superior 2020 (SIR Iber). Ediciones Profesionales de la Información. https://doi. org/10.3145/sir-iber-2020

Fernández, J.M. (2014). Cartas al editor. Anales Médicos de la Asociación Médico del Centro Médico ABC, 59(2), 84-85. https://www.medigraphic.com/cgi-bin/new/ resumen.cgi?IDARTICULO=50230\&id2=

Garred, P., Larsen, F., Seyfarth, J., Fujita, R., \& Madsen, H. O. (2006). Mannose-binding lectin and its genetic variants. Genes and Immunity, 7(2), 85 - 94. https:// doi.org/10.1038/sj.gene.6364283

Huamaní, C., \& Mayta-Tristán, P. (2010). Producción científica peruana en medicina y redes de colaboración, análisis del Science Citation Index 2000-2009. Revista Peruana de Medicina Experimental y Salud Publica, 27(3), 315-325. http://www. scielo.org.pe/scielo.php?script=sci_arttext $\&$ pi $\mathrm{d}=$ S1726-46342010000300003\&lng=es\&tlng=es

Jiménez, J.M. (2015). Tipos de publicaciones científicas. Orthotips AMOT, 11(2), 58 - 67. https://www.medigraphic.com/cgi-bin/new/resumen.cgi?IDARTICULO=59668 
Knowles, K. M., Paiva, L. L., Sanchez, S. E., Revilla, L., Lopez, T., Yasuda, M. B., Yanez, N. D., Gelaye, B., \& Williams, M. A. (2011). Waist Circumference, Body Mass Index, and Other Measures of Adiposity in Predicting Cardiovascular Disease Risk Factors among Peruvian Adults. International journal of hypertension, 2011, 931402. https://doi.org/10.4061/2011/931402

Limaymanta, C. H., Zulueta-Rafael, H., Restrepo-Arango, C., \& Alvarez-Muñoz, P. (2020). Análisis bibliométrico y cienciométrico de la producción científica de Perú y Ecuador desde Web of Science (2009-2018). Información, Cultura y Sociedad, 43, 31-52. https://doi. org/10.34096/ics.i43.7926

Miguel, S. (2011). Revistas y producción científica de América Latina y el Caribe: su visibilidad en SciELO, RedALyC y SCOPUS. Revista Interamericana de Bibliotecología, 34(2). https://revistas.udea.edu.co/index. php/RIB/article/view/10366

Ministerio de Educación (2020). Política Nacional de Educación Superior y Técnico - Productiva: Decreto Supremo N. ${ }^{\circ} 2012$ - 2020 - MINEDU. https://cdn. www.gob.pe/uploads/document/file/1481464/Política\%20Nacional\%20de\%20Educación\%20Superior\%20y\%20Técnico-Productiva.pdf

NCD Risk Factor Collaboration (NCD-RisC). (2019). Rising rural body-mass index is the main driver of the global obesity epidemic in adults. Nature, 569, 260-264. https://doi.org/10.1038/s41586-019-1171-x

Niño-Puello, M. (2013). El inglés y su importancia en la investigación científica: algunas reflexiones. Revista Colombiana de Ciencia Animal - RECIA, 5(1), 243254. https://doi.org/10.24188/recia.v5.n1.2013.487

Perilla-Rodríguez, L. M., \& Pérez-Acosta, A. M. (2016). Normalización de las firmas de autor y de institución: de los nombres a los ID. Avances en Psicología Latinoamericana, 34(3), 437-439. http://www. scielo.org.co/scielo.php?script=sci_arttext\&pi$\mathrm{d}=$ S1794-47242016000300001\&lng=en\&tlng=es.

Piedra, Y., \& Martínez, A. (2007). Producción científica. Ciencias de la Información, 38(3), 33 - 38. https://www. redalyc.org/articulo.oa?id=181414861004

Resolución del Consejo Directivo No 024-2017-SUNEDU/CD. "Resolución que otorga la licencia institucional a la
Universidad de San Martín de Porres, para ofrecer el servicio educativo superior universitario" (2017). https://filialnorte.usmp.edu.pe/pdf/convenio/resolucionLicenciamientoSUNEDU.pdf

Risco de Dominguez, G. (2007). Educación médica: nuevas tendencias, desafíos y oportunidades. Revista Digital de Investigación en Docencia Universitaria, 3(1), 1-20. https://doi.org/10.19083/ridu.3.20

Romero, R. (1995). Hipertensión portal y la circulación del bazo. Revista de gastroenterología del Perú, 15(1), 110 - 117. https://pesquisa.bvsalud.org/portal/resource/pt/lil-161912

Scimago Journal \& Country Rank (2021). Perú. https://www. scimagojr.com/countrysearch.php?country=PE

Solano, E., Castellanos, S., López, M.M., \& Hernández, J. (2009). La bibliometría: una herramienta eficaz para evaluar la actividad científica postgraduada. MediSur, 7(4), 59-62. http:// scielo.sld.cu/scielo.php?script=sci_arttext \&pi$\mathrm{d}=$ S1727-897X2009000400011\&lng=es\&tlng=es.

Superintendencia Nacional de Educación Superior Universitaria (2020). II Informe Bienal sobre la Realidad Universitaria en el Perú. https://www. gob.pe/institucion/sunedu/informes-publicaciones/1093280-ii-informe-bienal-sobre-la-realidad-universitaria-en-el-peru

Tarango, J., Hernández-Gutiérrez, P.Z., \& Vázquez-Guzmán, D. (2015). Evaluation of scientific production in Mexican state public universities (2007-2011) using principal component analysis. Profesional de la Información, 24(5), 567-576. https://doi.org/10.3145/ epi.2015.sep.06

Taype-Rondán, A, \& Luque, L. (2014). Producción científica en Scopus de la Universidad de San Martín de Porres, Lima, Perú. Horizonte Médico (Lima), 14(4), 37-42. http://www.scielo.org.pe/scielo.php?scrip$\mathrm{t}=$ sci_arttext\&pid=S1727-558X2014000400007\&ln $\mathrm{g}=\mathrm{es} \&$ tlng=es.

Vojvodic, Y., \& Marroquin, H. (1995). Sepsis intrabdominal: manejo quirúrgico. Revista de gastroenterología del Perú, 15(1), 62 - 73. https://sisbib.unmsm.edu.pe/ BVRevistas/gastro/Vol_15N1/sepsis_intrabdom.htm

RIDU / Revista Digital de Investigación en Docencia Universitaria / e-ISSN: 2223-2516

(C) Los autores. Este artículo es publicado por la Revista Digital de Investigación en Docencia Universitaria del Área de Institutional Research and Effectiveness de la Dirección de Aseguramiento de la Calidad, Universidad Peruana de Ciencias Aplicadas. . "Este es un artículo de acceso abierto, distribuido bajo los términos de la LicenciaCreativeCommons Atribución 4.0 Internacional (CC BY 4.0) (https://creativecommons.org/licenses/by/4.0/deed.es), que permite el uso, distribución y reproducción en cualquier medio, siempre que la obra original sea debidamente citada." 